Document downloaded from:

http://hdl.handle.net/10251/155868

This paper must be cited as:

Cebrian, S.; Perez, J.; Bandac, I.; Labarga, L.; Alvarez-Puerta, V.; Azevedo, CDR.; Benlloch-Rodriguez, JM.... (2017). Radiopurity assessment of the energy readout for the NEXT double beta decay experiment. Journal of Instrumentation. 12. https://doi.org/10.1088/1748-0221/12/08/T08003

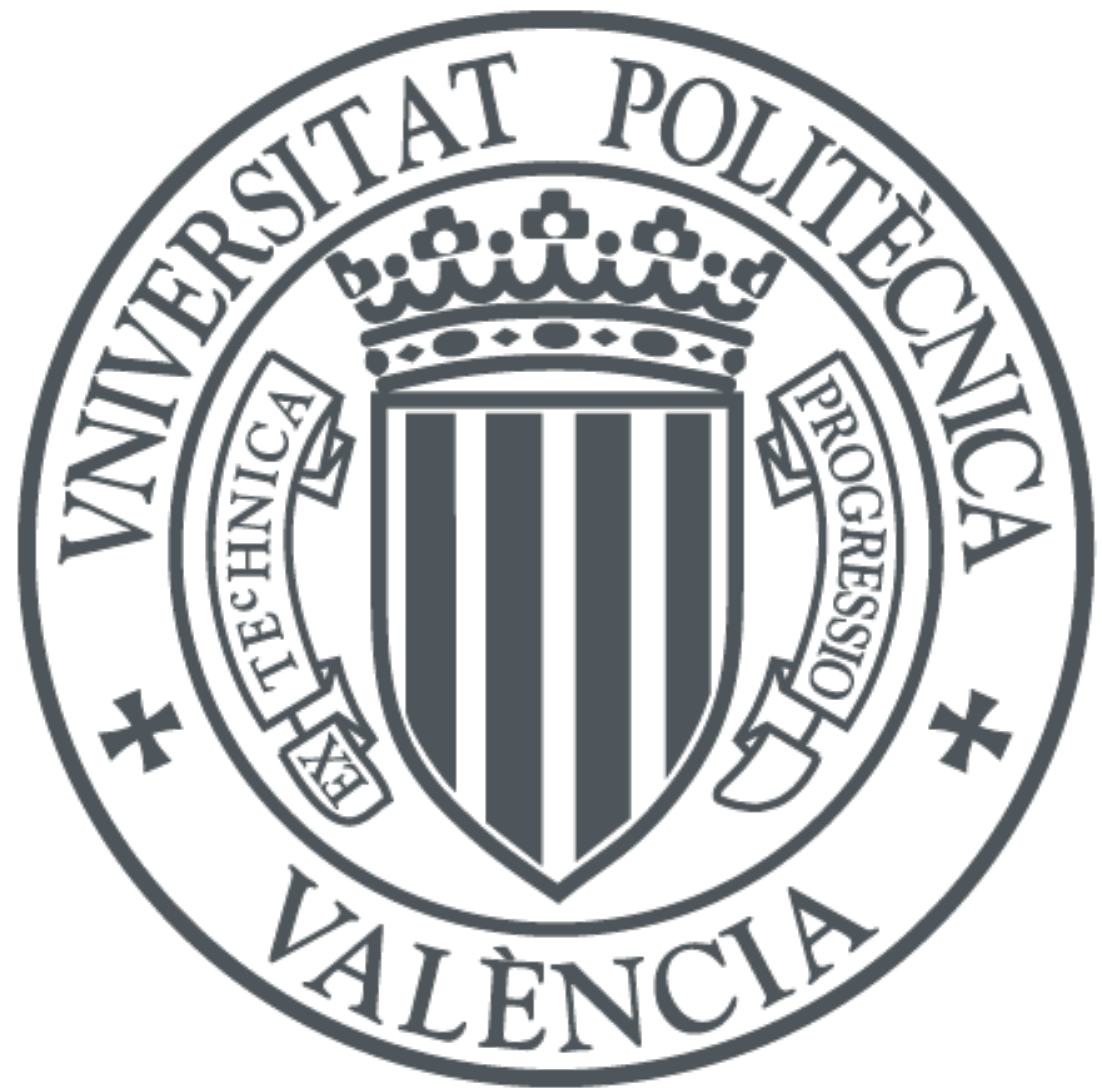

The final publication is available at

https://doi.org/10.1088/1748-0221/12/08/T08003

Copyright IOP Publishing

Additional Information 


\title{
Radiopurity assessment of the energy readout for the NEXT double beta decay experiment
}

\author{
S. Cebrián, ${ }^{a, b, 1}$ J. Pérez, ${ }^{c}$ I. Bandac, ${ }^{b}$ L. Labarga, ${ }^{d}$ V. Álvarez, ${ }^{c}$ C.D.R. Azevedo, ${ }^{e}$ \\ J.M. Benlloch-Rodríguez, ${ }^{c}$ F.I.G.M. Borges,${ }^{f}$ A. Botas, ${ }^{c}$ S. Cárcel, ${ }^{c}$ J.V. Carrión, ${ }^{c}$ \\ C.A.N. Conde,${ }^{f}$ J. Díaz, ${ }^{c}$ M. Diesburg, ${ }^{g}$ J. Escada,${ }^{f}$ R. Esteve, ${ }^{h}$ R. Felkai, ${ }^{c}$ L.M.P. Fernandes,${ }^{i}$ \\ P. Ferrario, ${ }^{c}$ A.L. Ferreira, ${ }^{e}$ E.D.C. Freitas, ${ }^{i}$ A. Goldschmidt, ${ }^{j}$ J.J. Gómez-Cadenas, ${ }^{c, 2}$ \\ D. González-Díaz, ${ }^{k}$ R.M. Gutiérrez, ${ }^{l}$ J. Hauptman, ${ }^{m}$ C.A.O. Henriques, ${ }^{i}$ A.I. Hernandez, ${ }^{l}$ \\ J.A. Hernando Morata, ${ }^{k}$ V. Herrero, ${ }^{h}$ B.J.P. Jones, ${ }^{n}$ A. Laing, ${ }^{c}$ P. Lebrun, ${ }^{g}$ I. Liubarsky, ${ }^{c}$
} N. López-March, ${ }^{c}$ M. Losada, ${ }^{l}$ J. Martín-Albo, ${ }^{c, 3}$ G. Martínez-Lema, ${ }^{k}$ A. Martínez, ${ }^{c}$ A.D. McDonald, ${ }^{n}$ F. Monrabal, ${ }^{n}$ C.M.B. Monteiro, ${ }^{i}$ F.J. Mora, ${ }^{h}$ L.M. Moutinho, ${ }^{e}$ J. Muñoz Vidal, ${ }^{c}$ M. Musti, ${ }^{c}$ M. Nebot-Guinot, ${ }^{c}$ P. Novella, ${ }^{c}$ D.R. Nygren ${ }^{n, 2}$ B. Palmeiro, ${ }^{c}$ A. Para, ${ }^{g}$ M. Querol, ${ }^{c}$ J. Renner, ${ }^{c}$ L. Ripoll, ${ }^{o}$ J. Rodríguez, ${ }^{c}$ L. Rogers, ${ }^{n}$ F.P. Santos,${ }^{f}$ J.M.F. dos Santos, ${ }^{i}$ A. Simón, ${ }^{c}$ C. Sofka, ${ }^{p, 4}$ M. Sorel, ${ }^{c}$ T. Stiegler, ${ }^{p}$ J.F. Toledo, ${ }^{h}$ J. Torrent, ${ }^{c}$ Z. Tsamalaidze, ${ }^{q}$ J.F.C.A. Veloso, ${ }^{e}$ J.A. Villar, ${ }^{a, b}$ R. Webb, ${ }^{p}$ J.T. White, ${ }^{p, 5}$ and N. Yahlali ${ }^{c}$

${ }^{a}$ Laboratorio de Física Nuclear y Astropartículas, Universidad de Zaragoza Calle Pedro Cerbuna, 12, 50009 Zaragoza, Spain

${ }^{b}$ Laboratorio Subterráneo de Canfranc Paseo de los Ayerbe s/n, 22880 Canfranc Estación, Huesca, Spain

${ }^{c}$ Instituto de Física Corpuscular (IFIC), CSIC \& Universitat de València Calle Catedrático José Beltrán, 2, 46980 Paterna, Valencia, Spain

${ }^{d}$ Departamento de Física Teórica, Universidad Autónoma de Madrid Campus de Cantoblanco, 28049 Madrid, Spain

${ }^{e}$ Institute of Nanostructures, Nanomodelling and Nanofabrication (i3N), Universidade de Aveiro Campus de Santiago, 3810-193 Aveiro, Portugal

${ }^{f}$ LIP, Department of Physics, University of Coimbra P-3004 516 Coimbra, Portugal

${ }^{g}$ Fermi National Accelerator Laboratory Batavia, Illinois 60510, USA

${ }^{h}$ Instituto de Instrumentación para Imagen Molecular (I3M), Centro Mixto CSIC -âĂŤ Universitat Politècnica de València Camino de Vera s/n, 46022 Valencia, Spain ${ }^{i}$ LIBPhys, Physics Department, University of Coimbra Rua Larga, 3004-516 Coimbra, Portugal

\footnotetext{
${ }^{1}$ Corresponding author. ${ }^{2}$ NEXT Co-spokesperson.

${ }^{3}$ Now at University of Oxford, United Kingdom. ${ }^{4}$ Now at University of Texas at Austin, USA.

${ }^{5}$ Deceased.
} 
${ }^{j}$ Lawrence Berkeley National Laboratory (LBNL)

1 Cyclotron Road, Berkeley, California 94720, USA

${ }^{k}$ Instituto Gallego de Física de Altas Energías, Univ. de Santiago de Compostela Campus sur, Rúa Xosé María Suárez Núñez, s/n, 15782 Santiago de Compostela, Spain

${ }^{l}$ Centro de Investigación en Ciencias Básicas y Aplicadas, Universidad Antonio Nariño

Sede Circunvalar, Carretera 3 Este No. 47 A-15, Bogotá, Colombia

${ }^{m}$ Department of Physics and Astronomy, Iowa State University

12 Physics Hall, Ames, Iowa 50011-3160, USA

${ }^{n}$ Department of Physics, University of Texas at Arlington

Arlington, Texas 76019, USA

${ }^{\circ}$ Escola Politècnica Superior, Universitat de Girona

Av. Montilivi, s/n, 17071 Girona, Spain

${ }^{p}$ Department of Physics and Astronomy, Texas A\&M University

College Station, Texas 77843-4242, USA

$q^{q}$ Joint Institute for Nuclear Research (JINR)

Joliot-Curie 6, 141980 Dubna, Russia

E-mail: scebrian@unizar.es

Aвstract: The "Neutrino Experiment with a Xenon Time-Projection Chamber" (NEXT) experiment intends to investigate the neutrinoless double beta decay of ${ }^{136} \mathrm{Xe}$, and therefore requires a severe suppression of potential backgrounds. An extensive material screening and selection process was undertaken to quantify the radioactivity of the materials used in the experiment. Separate energy and tracking readout planes using different sensors allow us to combine the measurement of the topological signature of the event for background discrimination with the energy resolution optimization. The design of radiopure readout planes, in direct contact with the gas detector medium, was especially challenging since the required components typically have activities too large for experiments demanding ultra-low background conditions. After studying the tracking plane, here the radiopurity control of the energy plane is presented, mainly based on gamma-ray spectroscopy using ultra-low background germanium detectors at the Laboratorio Subterráneo de Canfranc (Spain). All the available units of the selected model of photomultiplier have been screened together with most of the components for the bases, enclosures and windows. According to these results for the activity of the relevant radioisotopes, the selected components of the energy plane would give a contribution to the overall background level in the region of interest of at most $2.4 \times 10^{-4}$ counts $\mathrm{keV}^{-1} \mathrm{~kg}^{-1} \mathrm{y}^{-1}$, satisfying the sensitivity requirements of the NEXT experiment.

Keywords: Double beta decay; Time-Projection Chamber (TPC); Gamma detectors (HPGe); Search for radioactive material 


\section{Contents}

1 Introduction 1

2 Measurements 4

3 Results 4

3.1 Photomultipliers 8

3.2 PMT bases 9

$\begin{array}{ll}3.3 & \text { Windows, PMT enclosures and other components } \\ \end{array}$

$\begin{array}{lll}4 & \text { Conclusion } & 14\end{array}$

\section{Introduction}

Double beta decay is a very active research topic in Neutrino Physics. The observation of the neutrinoless mode, as a peak at the transition energy, would give unique information on the neutrino nature and mass (see for instance [1]-[4]). Since it is a very rare process, an ultra-low background level in the region where the signal is expected to appear is a must for this kind of experiment. NEXT ("Neutrino Experiment with a Xenon Time-Projection Chamber") [5] aims to search this in ${ }^{136}$ Xe at the Canfranc Underground Laboratory (Laboratorio Subterráneo de Canfranc, LSC) [6], located in the Spanish Pyrenees, with a source mass of $\sim 100 \mathrm{~kg}$ (NEXT-100 phase). The NEXT-100 detector is designed as an electroluminescent high-pressure xenon gas Time Projection Chamber (TPC) with two important features: very good energy resolution (better than 1\% FWHM at the transition energy of ${ }^{136} \mathrm{Xe}, \mathrm{Q}_{\beta \beta}=2.458 \mathrm{MeV}$ ) and topological reconstruction for the discrimination of signal and background events. As sketched in figure 1, light from the Xe electroluminescence generated at the anode is recorded both in the photosensor plane right behind it for tracking and in the plane behind the transparent cathode at the opposite side of the pressure vessel for a precise energy measurement. The separate energy and tracking readout planes use different sensors. Photomultiplier tubes (PMTs) are used for calorimetry, and for determining the start of the event thanks to the detection of the primary scintillation. Silicon photomultipliers (SiPMs) are used for tracking. After successful work on prototypes [7]-[17], the NEW (NEXT-WHITE) detector ${ }^{1}$ is fully operational at LSC. It is the first phase of the NEXT detector to operate underground; the NEW apparatus is a downscale 1:20 in mass of NEXT-100. A low background run using depleted Xe is foreseen during 2017; these data will be essential to validate the background model of NEXT-100, based on radiopurity measurements of all the relevant components, like the ones presented here.

As shown in [18], an excellent sensitivity is expected for NEXT-100. For a background rate of $4 \times 10^{-4}$ counts $\mathrm{keV}^{-1} \mathrm{~kg}^{-1} \mathrm{y}^{-1}$ in the energy region of interest, the experiment is sensitive to a neutrinoless decay half life up to $6 \times 10^{25}$ years after running for 3 effective years. The required

\footnotetext{
${ }^{1}$ The name honours the memory of the late Professor James White, key scientist of the NEXT project.
} 


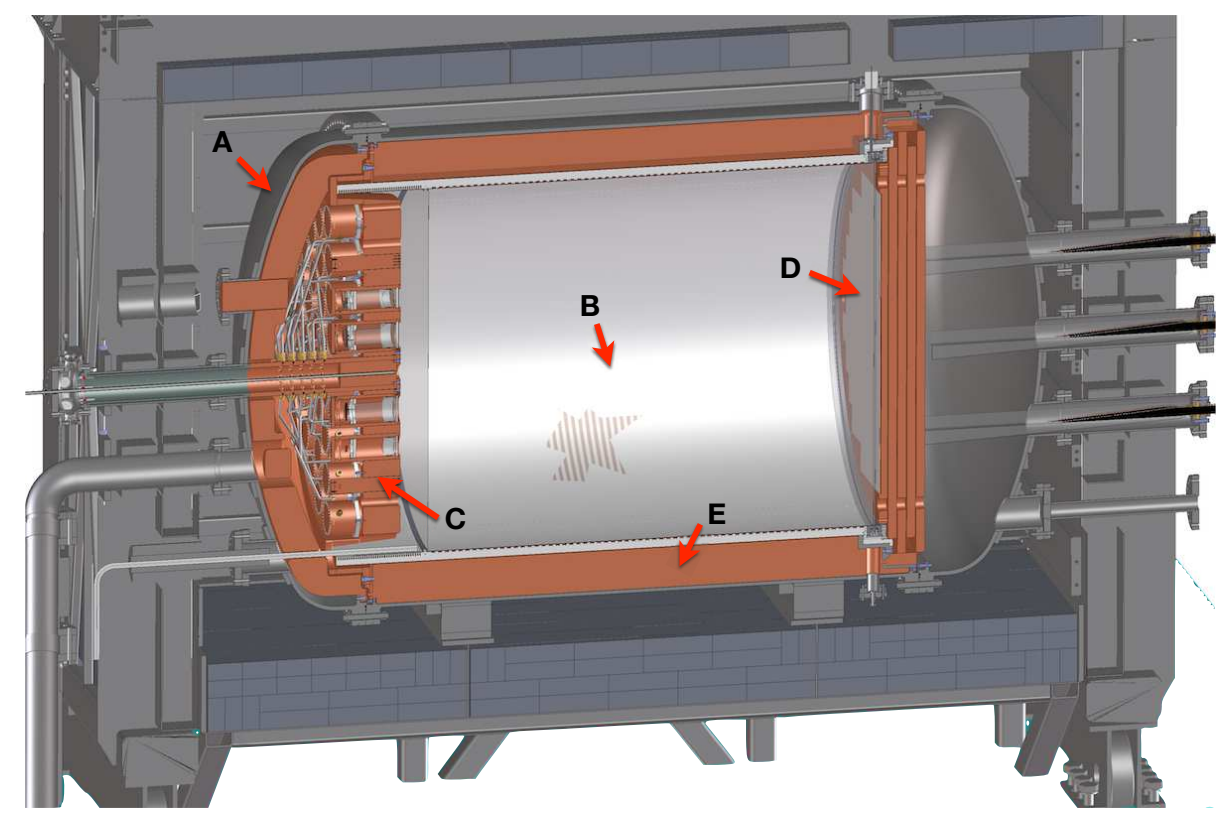

Figure 1. Cross-section view of the NEXT-100 detector inside its lead castle shield. A stainless steel pressure vessel (A) houses the electric-field cage (B) and the two sensor planes (energy plane, C; tracking plane, D) located at opposite ends of the chamber. The active volume is shielded from external radiation by at least $12 \mathrm{~cm}$ of copper $(\mathrm{E})$ in all directions.

background level is achievable thanks to passive shieldings, background discrimination techniques based on charged particle tracking $[17,19]$ and a thorough material radiopurity control. The most dangerous background sources are ${ }^{208} \mathrm{Tl}$ and ${ }^{214} \mathrm{Bi}$, isotopes of the progeny of ${ }^{232} \mathrm{Th}$ and ${ }^{238} \mathrm{U}$, because of their ability to generate a signal-like track in the fiducial volume with energy around $\mathrm{Q}_{\beta \beta}$.

A material screening and selection process for NEXT components has been underway for several years. Determination of the activity levels is based on gamma-ray spectroscopy using ultralow background germanium detectors at LSC and also on other techniques like Glow Discharge Mass Spectrometry (GDMS) and Inductively Coupled Plasma Mass Spectrometry (ICPMS). Materials to be used in the shielding, pressure vessel, electroluminescence and high voltage components, and energy and tracking readout planes have been measured and results have been presented in [20]-[23]. These results are the input for the construction of a precise background model of the NEXT experiment based on Monte Carlo simulations [18]. The design of radiopure readout planes is complicated by the fact that sensors, printed circuit boards and electronic components, involving typically different composite materials, show in many cases activity levels too large to be used in experiments demanding ultra-low background conditions (see for instance [24]-[26]). Exhaustive screening programs specifically for both the tracking and energy planes were undertaken; the former was presented in [27] and the latter is described here.

Figure 2 shows some drawings of the energy plane designed for the NEW detector. Following this design, the energy plane of NEXT-100 (see figure 1) will be composed of 60 Hamamatsu 


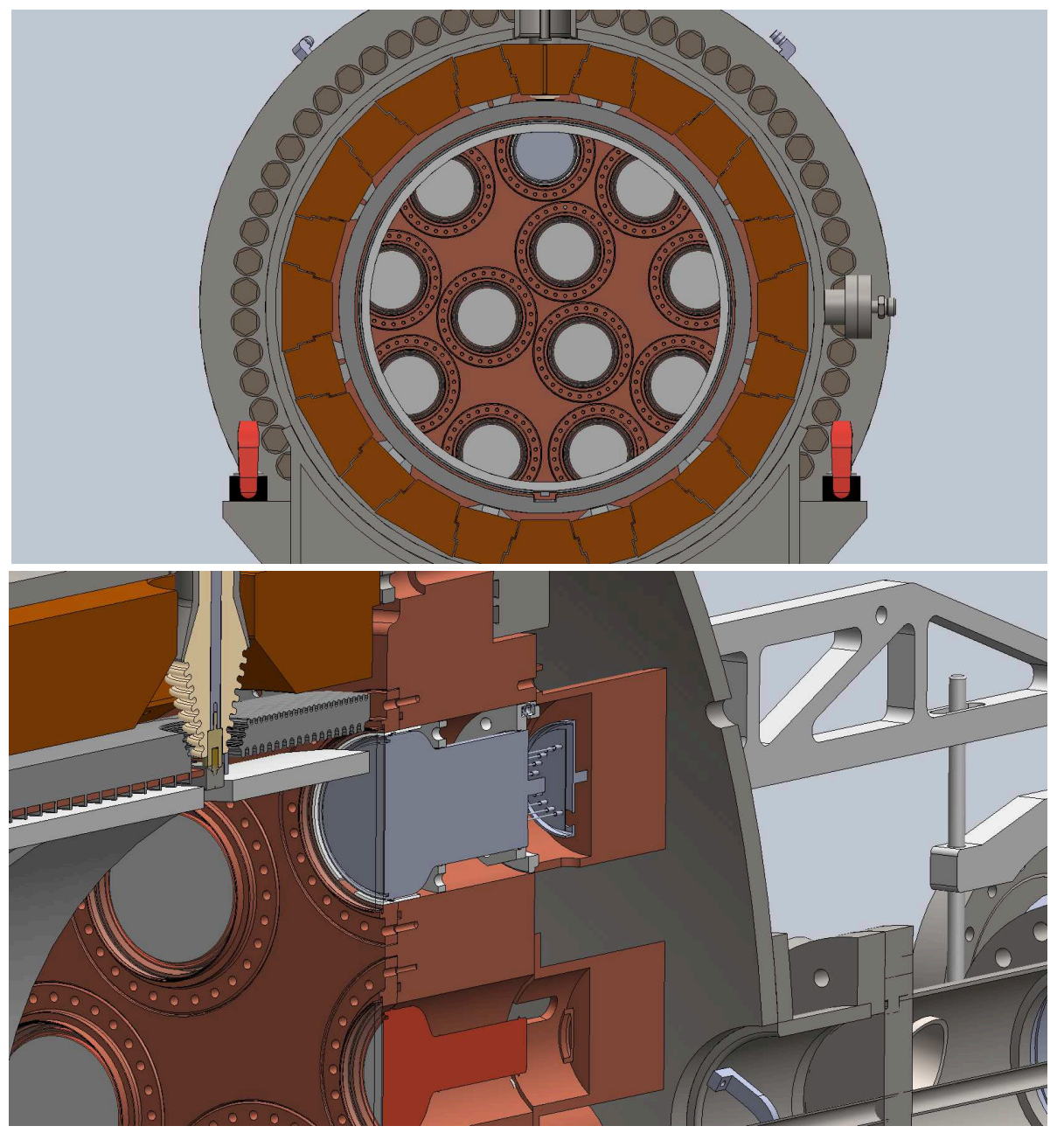

Figure 2. Design of the energy plane of the NEW detector. Top: View of the 12 PMTs and windows, including each one 28 bolts per flange, and the copper plate. Bottom: Zoomed image showing the PMT body (in red), the pins connecting the PMT body with the base, and the copper caps behind the PMT base.

R11410-10 photomultiplier tubes located behind the cathode of the TPC and covering approximately $30 \%$ of its area, as a compromise between the need to collect as much light as possible and the need to minimize the number of sensors to reduce cost, technical complexity and radioactivity. The selected model R11410-10 is a 3" PMT specially developed for low-background operation, equipped with a synthetic silica window and a photocathode made of low temperature bialkali with high quantum efficiency. The PMTs are optically coupled to sapphire windows using an optical gel with a proper refractive index. The external face of the windows is coated with tetraphenylbutadiene (TPB) to shift the xenon VUV light to blue. In NEW, the 12 PMTs used are sitting in an unique volume (either vacuum or nitrogen at 1 bar) separated from the xenon gas volume by a copper plate. Copper caps, having a thickness similar to that of the copper plate, are placed behind the PMT bases too.

The structure of the paper is the following. Section 2 summarizes all the measurements performed, describing both the samples analyzed and the detectors used. Activity results obtained 
are collected in section 3, together with the discussion of implications for design and for the NEXT-100 background model. Finally, conclusions are drawn in section 4.

\section{Measurements}

The material screening program of the energy readout of the NEXT experiment is mainly based on germanium gamma-ray spectrometry using ultra-low background detectors operated at a depth of 2450 m.w.e., from the Radiopurity Service of LSC; being a non-destructive technique, the actual components to be used in the experiment can be analyzed. Some complementary measurements have been made by GDMS, performed by Evans Analytical Group in France, providing concentrations of U, Th and K. The Radiopurity Service of LSC offers several detectors to measure ultra-low level radioactivity [28]. They are p-type closed-end coaxial 2.2-kg High Purity germanium detectors, from Canberra France, with aluminum or copper cryostats and relative efficiencies ${ }^{2}$ in the range from 100 to $110 \%$. Data acquisition is based on Canberra DSA 1000 modules and shielding consists of 5 or $10 \mathrm{~cm}$ of copper in the inner part surrounded by $20 \mathrm{~cm}$ of low activity lead, flushed with nitrogen gas to avoid airborne radon intrusion. The measurements related with the energy readout were carried out at LSC using different detectors (named GeAnayet, GeAspe, GeLatuca, GeOroel, GeTobazo and Obelix). The detection efficiency required to quantify activities was estimated by Monte Carlo simulations based on the Geant4 [29] code for each sample, accounting for intrinsic efficiency, the geometric factor and self-absorption at the sample; the uncertainty in this efficiency is estimated to be $10 \%$ and it is included in the calculation of the uncertainty for the derived activities. Activities of different sub-series in the natural chains of ${ }^{238} \mathrm{U},{ }^{232} \mathrm{Th}$ and ${ }^{235} \mathrm{U}$ as well as of common primordial, cosmogenic or anthropogenic radionuclides like ${ }^{40} \mathrm{~K},{ }^{60} \mathrm{Co}$ and ${ }^{137} \mathrm{Cs}$ have been evaluated by analyzing the most intense gamma lines of different isotopes; activities have been quantified when possible and upper limits with a $95.45 \%$ C.L. have been derived otherwise. More details on the detectors, their background counting rates, validation of efficiency estimates and the analysis procedure can be found at [20,27].

Table 1 summarizes the measurements performed for the samples analyzed in this work, indicating material and supplier, the detector used, the size of the sample and the live time of data taking. All the samples were cleaned in an ultrasonic bath and with pure alcohol before starting the screening, unless this could damage the component.

\section{Results}

The activity results obtained for the samples analyzed dealing with the energy readout plane are all summarized in table 2; reported errors correspond to $1 \sigma$ uncertainties including both statistical and efficiency uncertainties. In the following, each sample is described and the corresponding results discussed.

${ }^{2}$ Efficiency relative to a $3^{\prime \prime} \times 3^{\prime \prime} \mathrm{NaI}$ detector at $1332 \mathrm{keV}$ and for a distance of $25 \mathrm{~cm}$ between source and detector. 
Table 1. Information on measurements performed using the germanium detectors of the LSC Radiopurity Service: component and supplier, detector used, sample size (mass or number of units) and screening live time. The corresponding row number of table 2 where the activity values obtained for each sample are reported is also quoted. For the measurements of second row for PMT R11410-10, Hamamatsu, see explanations in section 3.1.

\begin{tabular}{lcccc}
\hline Component, Supplier & \# in table 2 & Detector & Sample size & Time (d) \\
\hline PMT R11410-10, Hamamatsu & 1 & GeAnayet & 1 unit & 33.7 \\
PMT R11410-10, Hamamatsu & 2 & GeAnayet & 3 units $\times 18$ runs & \\
\hline Capacitors $1.5 \mu \mathrm{F}$, AVX & 3 & GeLatuca & 392 units $(0.16 \mathrm{~g} / \mathrm{unit})$ & 37.8 \\
Capacitors $4.7 \mu \mathrm{F}$, AVX & 4 & GeAnayet & 156 units $(0.33 \mathrm{~g} / \mathrm{unit})$ & 28.0 \\
Polypropylene capacitors, Vishay & 5 & GeAspe & 46 units $(8 \mathrm{~g} / \mathrm{unit})$ & 22.5 \\
Resistors, Finechem & 6 & GeLatuca & 1200 units $(8.8 \mathrm{mg} / \mathrm{unit})$ & 38.5 \\
Resistors, KOA RS & 7 & GeTobazo & 100 units $(16.2 \mathrm{mg} / \mathrm{unit})$ & 32.2 \\
Resistors, Mouser & 8 & Obelix & 100 units $(9 \mathrm{mg} / \mathrm{unit})$ & 54.1 \\
Pin receptacles, Mill Max & 9 & GeLatuca & 1535 units $(51 \mathrm{mg} / \mathrm{unit})$ & 31.9 \\
Thermal epoxy, Electrolube & 10 & GeLatuca & $706 \mathrm{~g}$ & 40.4 \\
Epoxy 2011, Araldite & 11 & GeLatuca & $1712 \mathrm{~g}$ & 29.6 \\
Solder paste, Multicore & 12 & GeLatuca & $457 \mathrm{~g}$ & 44.3 \\
Kapton-Cu cable, Allectra & 13 & GeAspe & $352 \mathrm{~g}$ & 12.2 \\
Cuflon, Polyflon & 14 & GeOroel & $1876 \mathrm{~g}$ & 24.3 \\
Kapton substrate, Flexible Circuit & 15 & GeAnayet & 50 units $(0.61 \mathrm{~g} / \mathrm{unit})$ & 54.7 \\
\hline Windows, Precision Sapphire Technologies & 16 & GeAnayet & $527 \mathrm{~g}$ & 44.9 \\
Optical gel, Nye Lubricants & 17 & GeAnayet & $53.5 \mathrm{~g}$ & 58.3 \\
TPB, Sigma Aldrich & 18 & GeAnayet & $4.1 \mathrm{~g}$ & 38.3 \\
PEDOT:PSS, Aldrich Chemistry & 19 & GeAspe & $115 \mathrm{ml}$ & 77.1 \\
M4 screws (manual cleaning) & 23 & GeLatuca & 40 units $(2.4 \mathrm{~g} / \mathrm{unit})$ & 30.3 \\
M4 screws (Alconox cleaning) & 24 & GeLatuca & 267 units $(2.4 \mathrm{~g} / \mathrm{unit})$ & 56.6 \\
Vacuum grease, Apiezon M & 25 & GeAspe & $85.4 \mathrm{~g}$ & 44.4 \\
Copper CuA1, Lugand Aciers & 26 & GeOroel & $94 \mathrm{~kg}$ & 68.6 \\
CuSn braid, RS & 29 & GeAspe & $1875 \mathrm{~g}$ & 38.2 \\
\hline
\end{tabular}




\begin{tabular}{|c|c|c|c|c|c|c|c|c|c|c|c|}
\hline \# & Component & Technique & Unit & ${ }^{238} \mathbf{U}$ & ${ }^{226} \mathbf{R a}$ & ${ }^{232} \mathbf{T h}$ & ${ }^{228} \mathbf{T h}$ & ${ }^{235} \mathbf{U}$ & ${ }^{40} \mathbf{K}$ & ${ }^{60} \mathrm{Co}$ & ${ }^{137} \mathrm{Cs}$ \\
\hline 1 & PMT & $\mathrm{Ge}$ & $\mathrm{mBq} / \mathrm{PMT}$ & $<187$ & $<1.8$ & $<5.4$ & $<3.4$ & $<1.6$ & $<29$ & $2.82 \pm 0.27$ & $<0.6$ \\
\hline 2 & PMT & $\mathrm{Ge}$ & $\mathrm{mBq} / \mathrm{PMT}$ & $<69$ & $0.35 \pm 0.08$ & $<2.1$ & $0.53 \pm 0.12$ & $0.43 \pm 0.11$ & $12.1 \pm 1.6$ & $3.80 \pm 0.27$ & $<0.3$ \\
\hline 3 & Capacitors $1.5 \mu \mathrm{F}, \mathrm{AVX}$ & $\mathrm{Ge}$ & $\mu \mathrm{Bq} /$ unit & $<360$ & $72 \pm 3$ & $49 \pm 3$ & $38 \pm 2$ & & $71 \pm 9$ & $<1$ & $<1$ \\
\hline 4 & Capacitors $4.7 \mu \mathrm{F}, \mathrm{AVX}$ & $\mathrm{Ge}$ & $\mu \mathrm{Bq} /$ unit & $<900$ & $123 \pm 7$ & $95 \pm 7$ & $86 \pm 6$ & & $123 \pm 21$ & $<3$ & $<2$ \\
\hline 5 & Capacitors Vishay & $\mathrm{Ge}$ & $\mathrm{mBq} / \mathrm{unit}$ & $10.4 \pm 2.7$ & $5.29 \pm 0.25$ & $8.52 \pm 0.51$ & $8.75 \pm 0.49$ & & $5.29 \pm 0.57$ & $<0.036$ & $<0.043$ \\
\hline 6 & Resistors Finechem & $\mathrm{Ge}$ & $\mu \mathrm{Bq} / \mathrm{unit}$ & $85 \pm 23$ & $4.1 \pm 0.3$ & $5.6 \pm 0.5$ & $4.4 \pm 0.3$ & & $83.6 \pm 8.7$ & $<0.2$ & $104 \pm 11$ \\
\hline 7 & Resistors KOA RS & $\mathrm{Ge}$ & $\mu \mathrm{Bq} /$ unit & $<852$ & $<7.7$ & $<14$ & $<4.1$ & $<3.5$ & $<29$ & $<2.1$ & $<1.5$ \\
\hline 8 & Resistors Mouser & $\mathrm{Ge}$ & $\mu \mathrm{Bq} /$ unit & $<182$ & $<7.0$ & $5.3 \pm 1.5$ & $<8.0$ & $3.7 \pm 1.1$ & $<37$ & $<1.7$ & $<1.8$ \\
\hline 9 & Pin receptacles & $\mathrm{Ge}$ & $\mu \mathrm{Bq} /$ unit & $217 \pm 42$ & $<1.1$ & $5.6 \pm 0.5$ & $4.5 \pm 0.4$ & $6.1 \pm 0.5$ & $20.5 \pm 2.4$ & $<0.3$ & $<0.2$ \\
\hline 10 & Thermal epoxy & $\mathrm{Ge}$ & $\mathrm{mBq} / \mathrm{kg}$ & $(1.0 \pm 0.2) 10^{3}$ & $169.4 \pm 7.9$ & $52.1 \pm 3.7$ & $54.4 \pm 3.2$ & & $105 \pm 12$ & $<1.1$ & $<1.3$ \\
\hline 11 & Epoxy Araldite & $\mathrm{Ge}$ & $\mathrm{mBq} / \mathrm{kg}$ & $<182$ & $<1.4$ & $<3.7$ & $<2.5$ & $<0.8$ & $15.0 \pm 2.4$ & $<0.4$ & $<0.4$ \\
\hline 12 & Solder paste & $\mathrm{Ge}$ & $\mathrm{mBq} / \mathrm{kg}$ & $<310$ & $<2.7$ & $<4.7$ & $<2.5$ & $<5.2$ & $<13$ & $<1.0$ & $<1.6$ \\
\hline 13 & Kapton-Cu cable & $\mathrm{Ge}$ & $\mathrm{mBq} / \mathrm{kg}$ & $<1.1 \times 10^{3}$ & $46.8 \pm 3.3$ & $<40$ & $<32$ & & $166 \pm 27$ & $<5.2$ & $<4.4$ \\
\hline 14 & Cuflon & $\mathrm{Ge}$ & $\mathrm{mBq} / \mathrm{kg}$ & $<33$ & $<1.3$ & $<1.1$ & $<1.1$ & $<0.6$ & $4.8 \pm 1.1$ & $<0.3$ & $<0.3$ \\
\hline 15 & Kapton substrate & $\mathrm{Ge}$ & $\mu \mathrm{Bq} /$ unit & $<2.8 \times 10^{3}$ & $<23$ & $77 \pm 13$ & $43.9 \pm 7.2$ & $<18$ & $<216$ & $<6.4$ & $<6.7$ \\
\hline 16 & Sapphire windows & $\mathrm{Ge}$ & $\mathrm{mBq} / \mathrm{kg}$ & $<275$ & $<2.7$ & $<7.6$ & $<5.5$ & $<2.1$ & $<18$ & $<0.7$ & $<1.0$ \\
\hline 17 & Optical gel & $\mathrm{Ge}$ & $\mathrm{mBq} / \mathrm{kg}$ & $<1.7 \times 10^{3}$ & $<22$ & $<49$ & $<18$ & $<16$ & $<173$ & $<4.5$ & $<5.8$ \\
\hline 18 & TPB & $\mathrm{Ge}$ & $\mathrm{Bq} / \mathrm{kg}$ & $<23$ & $<0.17$ & $<0.57$ & $<0.15$ & $<0.11$ & $<1.7$ & $<0.05$ & $<0.05$ \\
\hline 19 & PEDOT:PSS & $\mathrm{Ge}$ & $\mu \mathrm{Bq} / \mathrm{ml}$ & $<626$ & $<6.9$ & $<23$ & $<4.8$ & $<3.9$ & $49 \pm 11$ & $<1.7$ & $<1.8$ \\
\hline 20 & Brazing paste & GDMS & $\mu \mathrm{Bq} / \mathrm{kg}$ & $55 \pm 10$ & & $49 \pm 4$ & & & $<31$ & & \\
\hline 21 & Brass bolts & GDMS & $\mu \mathrm{Bq} / \mathrm{kg}$ & $8.9 \pm 0.7$ & & $6.9 \pm 0.2$ & & & $<31$ & & \\
\hline 22 & SS screws & GDMS & $\mathrm{mBq} / \mathrm{kg}$ & $3.25 \pm 0.25$ & & $0.57 \pm 0.08$ & & & $<0.19$ & & \\
\hline 23 & M4 screws, manual clean & $\mathrm{Ge}$ & $\mu \mathrm{Bq} /$ unit & $<2.2 \times 10^{3}$ & $<21$ & $<60$ & $20.0 \pm 4.6$ & $<12$ & $<93$ & $14.0 \pm 1.8$ & $<6.0$ \\
\hline 24 & M4 screws, Alconox clean & $\mathrm{Ge}$ & $\mu \mathrm{Bq} /$ unit & $<616$ & $<8.6$ & $14.9 \pm 3.4$ & $17.4 \pm 1.8$ & $3.7 \pm 1.0$ & $<19$ & $13.4 \pm 1.1$ & $<1.4$ \\
\hline 25 & Vacuum grease & $\mathrm{Ge}$ & $\mathrm{mBq} / \mathrm{kg}$ & $<1.0 \times 10^{3}$ & $<10$ & $<43$ & $<8.5$ & $<6.1$ & $<49$ & $<3.5$ & $<2.9$ \\
\hline 26 & $\mathrm{CuA} 1$ & $\mathrm{Ge}$ & $\mathrm{mBq} / \mathrm{kg}$ & $<4.1$ & $<0.16$ & $<0.15$ & $<0.13$ & $<0.17$ & $<0.37$ & $0.04 \pm 0.01$ & $<0.04$ \\
\hline 27 & $\mathrm{CuA} 1$ & GDMS & $\mu \mathrm{Bq} / \mathrm{kg}$ & $<12$ & & $<4.1$ & & & 62 & & \\
\hline 28 & $\mathrm{CuC1}$ & GDMS & $\mu \mathrm{Bq} / \mathrm{kg}$ & $25 \pm 5$ & & $15 \pm 4$ & & & 190 & & \\
\hline 29 & CuSn braid & $\mathrm{Ge}$ & $\mathrm{mBq} / \mathrm{kg}$ & $<168$ & $<2.4$ & $<7.1$ & $<2.1$ & $<1.8$ & $<14$ & $<0.6$ & $<0.5$ \\
\hline
\end{tabular}

(Follows at next page) 
(Continuation)

\# Component

Technique Unit

${ }^{238} \mathbf{U}$

${ }^{226} \mathbf{R a}$

${ }^{232}$ Th

${ }^{228} \mathbf{T h}$

${ }^{235} \mathbf{U}$

${ }^{40} \mathbf{K}$

${ }^{60} \mathrm{Co}$

Table 2: Activities measured for the energy readout components used in NEXT.

Results reported for ${ }^{238} \mathrm{U}$ and ${ }^{232} \mathrm{Th}$ correspond to the upper part of the chains

and those of ${ }^{226} \mathrm{Ra}$ and ${ }^{228} \mathrm{Th}$ give activities of the lower parts. For component

\#2, see explanations in section 3.1. 
Table 3. Activities (in $\mathrm{mBq}$ ) measured at each individual run screening altogether three different PMTs per run. Results reported for ${ }^{238} \mathrm{U}$ and ${ }^{232} \mathrm{Th}$ correspond to the upper part of the chains and those of ${ }^{226} \mathrm{Ra}$ and ${ }^{228}$ Th give activities of the lower parts.

\begin{tabular}{lcccccccccc}
\hline Run & $\begin{array}{c}\text { Time } \\
\text { (d) }\end{array}$ & ${ }^{238} \mathrm{U}$ & ${ }^{226} \mathrm{Ra}$ & ${ }^{232} \mathrm{Th}$ & ${ }^{228} \mathrm{Th}$ & ${ }^{235} \mathrm{U}$ & ${ }^{40} \mathrm{~K}$ & ${ }^{60} \mathrm{Co}$ & ${ }^{137} \mathrm{Cs}$ & ${ }^{54} \mathrm{Mn}$ \\
\hline 1 & 30.6 & $<266$ & $<2.7$ & $<7.8$ & $<6.1$ & $<3.4$ & $30.4 \pm 8.1$ & $12.2 \pm 1.0$ & $<1.0$ & $1.1 \pm 0.3$ \\
2 & 20.2 & $<340$ & $<3.3$ & $<9.1$ & $<7.9$ & $<4.4$ & $<60$ & $11.1 \pm 0.9$ & $<1.3$ & $<1.7$ \\
3 & 22.0 & $<320$ & $<3.3$ & $<10$ & $<7.3$ & $<3.8$ & $39.5 \pm 9.4$ & $10.4 \pm 0.9$ & $<1.2$ & $<1.3$ \\
4 & 18.3 & $<351$ & $<4.3$ & $<9.5$ & $<9.6$ & $<3.8$ & $41 \pm 10$ & $11.5 \pm 1.0$ & $<1.4$ & $1.1 \pm 0.3$ \\
5 & 41.0 & $<229$ & $<2.5$ & $<6.6$ & $<7.2$ & $<3.1$ & $32.3 \pm 7.7$ & $10.8 \pm 0.8$ & $<0.8$ & $1.1 \pm 0.2$ \\
6 & 39.8 & $<232$ & $<2.7$ & $<9.3$ & $<6.2$ & $<3.1$ & $30.0 \pm 7.5$ & $10.4 \pm 0.8$ & $<0.8$ & $1.0 \pm 0.3$ \\
7 & 23.0 & $<317$ & $<4.3$ & $<9.1$ & $<6.5$ & $<2.6$ & $34.2 \pm 8.9$ & $10.9 \pm 0.9$ & $<1.1$ & $<1.4$ \\
8 & 38.7 & $<234$ & $<3.0$ & $<9.3$ & $<5.9$ & $<3.2$ & $35.5 \pm 7.9$ & $12.3 \pm 1.0$ & $<0.8$ & $<1.2$ \\
9 & 19.5 & $<330$ & $<3.4$ & $<9.2$ & $<10$ & $<4.3$ & $34.8 \pm 9.5$ & $11.3 \pm 0.9$ & $<1.2$ & $<1.8$ \\
10 & 27.8 & $<293$ & $<3.9$ & $<9.4$ & $<6.3$ & $<4.4$ & $36.9 \pm 8.6$ & $12.0 \pm 0.9$ & $<1.0$ & $<1.4$ \\
11 & 30.4 & $<286$ & $2.7 \pm 0.8$ & $<11$ & $<6.8$ & $<2.2$ & $38.0 \pm 8.7$ & $11.1 \pm 0.9$ & $<1.3$ & $<1.4$ \\
12 & 33.5 & $<405$ & $<3.2$ & $<6.9$ & $<6.5$ & $<3.8$ & $28.3 \pm 7.9$ & $11.0 \pm 0.9$ & $<0.8$ & $0.9 \pm 0.3$ \\
13 & 23.6 & $<361$ & $<5.2$ & $<8.3$ & $<8.7$ & $2.5 \pm 0.7$ & $50 \pm 10$ & $11.4 \pm 0.9$ & $<1.1$ & $1.2 \pm 0.3$ \\
14 & 38.6 & $<337$ & $<3.7$ & $<8.9$ & $<6.9$ & $<3.0$ & $30.7 \pm 7.8$ & $11.7 \pm 0.9$ & $<0.8$ & $0.9 \pm 0.2$ \\
15 & 24.2 & $<470$ & $3.0 \pm 0.9$ & $<8.6$ & $<6.2$ & $<4.3$ & $34.5 \pm 9.0$ & $10.3 \pm 0.8$ & $<1.2$ & $<1.4$ \\
16 & 24.1 & $<418$ & $<4.5$ & $<11$ & $<7.9$ & $<4.1$ & $41.6 \pm 9.4$ & $12.2 \pm 1.0$ & $<1.1$ & $0.8 \pm 0.2$ \\
17 & 19.3 & $<443$ & $<4.9$ & $<10$ & $<8.6$ & $<3.5$ & $<61$ & $12.1 \pm 1.0$ & $<1.2$ & $<1.7$ \\
18 & 23.6 & $<448$ & $<5.6$ & $<8.8$ & $<9.0$ & $<2.6$ & $45.3 \pm 9.3$ & $12.5 \pm 1.0$ & $<1.0$ & $<1.1$ \\
\hline All & 498.2 & $<83$ & $1.05 \pm 0.24$ & $<2.8$ & $1.58 \pm 0.35$ & $1.30 \pm 0.32$ & $36.3 \pm 4.8$ & $11.40 \pm 0.81$ & $<0.35$ & \\
\hline & & & & & & & & & &
\end{tabular}

\subsection{Photomultipliers}

The photomultiplier tubes are the basic element of the energy readout plane of NEXT. The number of required PMTs is 12 for NEW and 60 for NEXT-100. All the 55 available units of the selected model, Hamamatsu ${ }^{3}$ R11410-10, have been screened using the same germanium detector (GeAnayet) at LSC during 2013, 2014 and 2015. First, a single photomultiplier was analyzed (row \#1 of table 2) and then eighteen runs were carried out, each one with three different units placed around the detector on a teflon support; data taking at each run ranged from 18.3 to 41.0 days per run and several reference backgrounds were measured in between. For the detection efficiency simulation, emissions are assumed to be uniformly generated in the kovar PMT enclosure. Activities for the three PMTs at each independent measurement were deduced and are summarized in table 3. The results obtained from different runs are roughly compatible; ${ }^{60} \mathrm{Co}$ activity has been always quantified, those of ${ }^{40} \mathrm{~K}$ and ${ }^{54} \mathrm{Mn}$ only in the most sensitive runs while upper limits have been obtained in general for all the other common isotopes.

Since, following these results, it seemed that the activity levels of all the screened PMTs

${ }^{3}$ http://www.hamamatsu.com 
were similar, a joint analysis of the available data was attempted in order to further increase the sensitivity by combining data from the eighteen independent runs performed with three PMTs altogether corresponding to a total exposure of 498.2 days. The obtained results are shown in last row of table 3. Since it was assumed that all the PMTs were equivalent, activity values per PMT were estimated just considering one third of the net signal measured; these results are shown in row \#2 of table 2. Thanks to this joint analysis, the activity of ${ }^{235} \mathrm{U}$ has been properly evaluated. Although there is also a clear net signal from ${ }^{54} \mathrm{Mn}$, a direct quantification of the activity has not been attempted since its half-life (312.3 days) is comparable to the time span of the measurements and then its decay should be properly taken into consideration. Concerning the lower parts of the ${ }^{232} \mathrm{Th}$ and ${ }^{238} \mathrm{U}$ chains, several lines show an excess of events above background statistically significant thanks to the accumulation of data; therefore, it has been possible to quantify the activity of some of their isotopes and a robust estimate of the average activity per PMT of ${ }^{226} \mathrm{Ra}$ and ${ }^{228} \mathrm{Th}$ has been achieved.

The same model of PMT has been screened for other experiments [30-32] and our results are in very good agreement with those found by XENON; in particular, ${ }^{60} \mathrm{Co}$ and ${ }^{40} \mathrm{~K}$ activities are virtually the same.

- The XENON1t collaboration has carried out a deep study of the radioactivity of the new PMT version Hamamatsu R11410-21 [33], based on analysis using GDMS and germanium spectrometry of individual components and units $[34,35]$. The main differences with respect to the version R11410-10 are the use of Co-free kovar body and high purity (instead of standard purity) Al seal. Comparing results for the two versions, it can be concluded that ${ }^{40} \mathrm{~K}$ and ${ }^{235} \mathrm{U}$ activities are comparable, ${ }^{60} \mathrm{Co}$ has been reduced about a factor $4-5$ in the new version and for the lower parts of the natural chains of ${ }^{232} \mathrm{Th}$ and ${ }^{238} \mathrm{U}$, activity is at the same level, about one-half mBq/PMT.

- Results for the version R11410-20 have been presented by the LUX-ZEPLIN collaboration [36]. Intrinsic radioactivity of the component materials to be used in the manufacture has been performed using several germanium detectors and upper limits set for the total activity of all components are comparable to the best quantified activities for other versions; only the upper limit set for ${ }^{40} \mathrm{~K}$ is a factor $\sim 4$ lower. The screening of final tubes is underway.

\subsection{PMT bases}

Each PMT base in the NEW set-up is composed of a total of 19 resistors of different electrical resistance, 7 capacitors (5 having a capacitance of $1.5 \mu \mathrm{F}$ and 2 with $4.7 \mu \mathrm{F}$ ) and 18 pin receptacles fixed on a kapton substrate using epoxy, a copper cap with a mass of $\sim 50 \mathrm{~g}$ and a 1-m-long cable made of kapton and copper. A paste was used for soldering. All these components used in the PMT bases have been separately screened; the PMT base design reflects a careful compromise between performance of electronics response (particularly concerning charge linearity) and radio purity.

Base capacitors are Tantalum Solid Electrolytic Chip Capacitors with Conductive Polymer Electrode, TCJ Series, supplied by AVX ${ }^{4}$. Two samples of units with the different capacitance and different size and mass have been screened. It was possible to quantify the activities from ${ }^{40} \mathrm{~K}$,

${ }^{4}$ http://www.avx.com 
${ }^{232} \mathrm{Th}$ and the lower part of the ${ }^{238} \mathrm{U}$ chain (rows \#3 and 4 of table 2). The measured activities in the larger capacitors are roughly a factor of 2 higher than in the smaller ones, which is also the ratio between the masses of each unit. The presence of ${ }^{182} \mathrm{Ta}$ (beta emitter with $\mathrm{Q}=1814.3 \mathrm{keV}$, $\mathrm{T}_{1 / 2}=114.6$ days, produced by neutron activation on ${ }^{181} \mathrm{Ta}$ ) was identified by means of several of its gamma emissions. In addition, a sample of capacitors from Vishay 5 (Metallized Polypropylene Film Capacitors, $5 \mu \mathrm{F}, 32 \times 11 \times 21 \mathrm{~mm}^{3}$ each unit) having the dielectric made of polypropylene was screened too. All common radioisotopes were quantified with activities of a few $\mathrm{mBq} / \mathrm{unit}$ (row \#5 of table 2), which are unacceptable for NEXT, and consequently the use of these polypropylene capacitors was discontinued.

SMD resistors to be used at the voltage divider from several suppliers have been screened:

- SM2 resistors supplied by the Japanese company Finechem ${ }^{6}$ have an alumina ceramic substrate. The dimensions of each unit are $3.2 \times 1.6 \times 0.55 \mathrm{~mm}^{3}$. Activities have been derived for ${ }^{40} \mathrm{~K}$ as well as for the ${ }^{232} \mathrm{Th}$ and ${ }^{238} \mathrm{U}$ chains (row \#6 of table 2). In addition, the resistors showed important activities from ${ }^{134} \mathrm{Cs}$ and ${ }^{137} \mathrm{Cs}$, which could be related to the Fukushima accident. For ${ }^{134} \mathrm{Cs}$ (beta emitter with $\mathrm{Q}=2058.98 \mathrm{keV}, \mathrm{T}_{1 / 2}=2.06 \mathrm{y}$ ), activity was $32.7 \pm 1.6 \mu \mathrm{Bq} / \mathrm{unit}$. The results obtained can be compared with the ones for SM5D Finechem resistors, showing no Cs activity, presented at [20,30]; results are roughly consistent taking into account that the volume of SM5 resistors is four times the one of SM2 resistors.

- Another sample consisted of resistors produced by KOA Speer ${ }^{7}$ and supplied by RS (Thin Film 1206, $62 \Omega$ ). Dimensions of each unit are $3.2 \times 1.6 \times 0.6 \mathrm{~mm}^{3}$. None of the common radioisotopes has been quantified and upper limits to their activities have been set (row \#7 of table 2).

- Finally, resistors from Mouser ${ }^{8}(62 \Omega)$ were analyzed too. In this case, activity of some isotopes has been quantified and upper limits for the other ones have been set (row \#8 of table 2).

Comparing the results from the three considered resistors, it can be concluded that the quantified activities or upper limits are at similar levels of a few $\mu \mathrm{Bq} / \mathrm{unit}$ for all of them; finally, 14 units from Finechem and 5 from RS have been selected.

Pin receptacles from Mill Max 9 (model 0327-0-15-15-34-27-10-0) having a shell made of brass alloy 360 were screened. Activities for most of the common radioisotopes have been quantified (row \#9 of table 2).

Thermally conductive epoxy produced by Electrolube ${ }^{10}$ (division of HK WENTWORTH LTD) was screened to be used on the PMT bases to dissipate heat. To prepare the sample the epoxy (EER2074A) and corresponding hardener (EER2074B) were mixed inside a clean container following specifications. A sample of the epoxy Araldite $2011^{11}$ was screened too. A mixture of

\footnotetext{
${ }^{5}$ http://www.vishay.com

${ }^{6} \mathrm{http}: / /$ www.jfine.co.jp

${ }^{7} \mathrm{http}: / /$ www.koaspeer.com

${ }^{8} \mathrm{http}: / /$ www.mouser.com

${ }^{9} \mathrm{http}: / / \mathrm{www} \cdot \mathrm{mill}-\mathrm{max} \cdot \mathrm{com}$

${ }^{10} \mathrm{http}: / / \mathrm{www}$. electrolube.com

${ }^{11} \mathrm{http} / / / \mathrm{www} . g 0-$ araldite.com
} 
Araldite2011-A (resin) and Araldite 2011-B (hardener) was prepared. As shown in rows \#10-11 of table 2 , Araldite epoxy showed a better radiopurity since only ${ }^{40} \mathrm{~K}$ was quantified and upper limits for the other common radioisotopes were derived; these are more stringent than those available from [37]. Therefore, the epoxy Araldite 2011 was finally used in the PMT bases.

A sample of lead-free SnAgCu solder paste supplied by Multicore (ref. 698840) was screened and results are presented in row \#12 of table 2. ${ }^{108 m} \mathrm{Ag}$, induced by neutron interactions and having a half-life of $\mathrm{T}_{1 / 2}=438 \mathrm{y}$, has been identified in the paste, with an activity of $(5.26 \pm 0.40) \mathrm{mBq} / \mathrm{kg}$, while upper limits of a few $\mathrm{mBq} / \mathrm{kg}$ have been set for the common radioactive isotopes.

A roll of the kapton-copper cable supplied by Allectra company ${ }^{12}$ was screened. Activities of some isotopes were quantified (row \#13 of table 2) and in addition, the presence of ${ }^{108 m} \mathrm{Ag}$ can be reported through the identification of its most intense gamma lines.

Concerning the base substrate, cuflon and kapton have been considered. Cuflon $\AA$ offers low activity levels, as shown in the measurement of samples from Crane Polyflon ${ }^{13}$ by GERDA [38] and at [39], using both ICPMS and Ge gamma spectroscopy. As presented in [20], a measurement of Polyflon cuflon made of a 3.18-mm-thick PTFE layer sandwiched by two 35- $\mu$ m-thick copper sheets was made for NEXT and results are shown in row \#14 of table 2 . Only activity of ${ }^{40} \mathrm{~K}$ could be quantified. Although cuflon could have been used too, kapton was finally selected and a sample of the produced 0.5 -mm-thick base substrates by Flexible Circuit ${ }^{14}$ was screened. Upper limits were set for all common radioisotopes except for ${ }^{232} \mathrm{Th}$ (row \#15 of table 2).

\subsection{Windows, PMT enclosures and other components}

Other components also used in the energy readout plane have been taken into consideration. Four sapphire crystals to be used as PMT windows were screened; each crystal was $6 \mathrm{~mm}$ thick with a diameter of $83.8 \mathrm{~mm}$. They were measured on a teflon support for protection. No isotope was quantified (row \#16 of table 2). Since the upper limits obtained from germanium spectrometry are quite high, results from Neutron Activation Analysis (NAA) presented at measurement \#155 in [40] by the EXO collaboration have been considered for the moment in the development of the NEXT-100 background model.

The silicone-based optical gel from Nye Lubricants Inc. ${ }^{15}$ (SmartGel NyoGel OCK-451), used for PMT coupling, was screened. The optical fluid and thickening agent were mixed in the clean room of LSC and left there for 26 hours in order to form a solid disk. The sample was prepared on a clean container. No isotope was quantified and upper limits were set for all of them (row \#17 of table 2). The quantity to be used per window is estimated to be about $2 \mathrm{~g}$.

A sample of the TPB material coating the enclosure windows, supplied by Sigma Aldrich ${ }^{16}$, was analyzed. The powder was prepared inside a clean Petri dish. Upper limits on the specific activity were set for all the radioisotopes (row \#18 of table 2). Due to the small mass of our sample, better results for this material from the same supplier can be found at [41, 42]; activities for ${ }^{238} \mathrm{U}$ and ${ }^{232} \mathrm{Th}$ at the level of tenths of $\mathrm{mBq} / \mathrm{kg}$ or even lower are reported there.

\footnotetext{
${ }^{12} \mathrm{http}: / /$ www.allectra.com

${ }^{13} \mathrm{http}: / /$ www.polyflon.com

${ }^{14} \mathrm{http}: / /$ www.flexiblecircuit.com

${ }^{15} \mathrm{http} / / / \mathrm{www}$.nyelubricants.com

${ }^{16} \mathrm{http}: / /$ www.sigmaaldrich.com
} 
A sample of PEDOT:PSS (1.3 wt\% dispersion in water) also from Aldrich Chemistry to be used as conductive coating on sapphire windows was screened too (row \#19 of table 2); only the activity of ${ }^{40} \mathrm{~K}$ could be quantified. It is applied by spin-coating and then dried to evaporate water, resulting in a $\sim 100$-nm-thick layer.

Other materials or components to be used at the PMT enclosures were analyzed by GDMS. A sample of brazing paste made of $72 \% \mathrm{Ag}$ and $28 \% \mathrm{Cu}$ with dimensions $12 \times 12 \times 12 \mathrm{~mm}^{3}$ was measured quantifying the $U$ and Th content (row \#20 of table 2). M4 vented screws made of 316 stainless steel were screened; the mass of each 2-cm-long unit is $2.32 \mathrm{~g}$. A sample of a M4 bolt made of brass, with length $22.65 \mathrm{~mm}$ and mass $3.08 \mathrm{~g}$, was also analyzed and the results are shown in rows \#21-22 of table 2. Since 28 units are needed per PMT can, brass bolts were preferred instead of the vented screws from the radiopurity point of view.

However, in principle, stainless steel has been used for mechanical reasons in the NEW set-up. Samples of M4 screws were screened using germanium detectors. Since these screws were pregreased, a cleaning procedure was necessary to remove the grease, which could affect the purity of the xenon gas and is expected to be non-radiopure; two options were analyzed. A manual cleaning was applied to a sample, by wiping the screws several times with alcohol by hand, cleaning them in an ultrasound bath with soap and afterwards rinsing with alcohol. For another sample of screws, cleaning was made using Alconox ${ }^{17}$ detergent 8 (5\% solution in water) in an ultrasound bath. Activities from ${ }^{60} \mathrm{Co},{ }^{235} \mathrm{U}$ and for the ${ }^{232} \mathrm{Th}$ chain have been quantified (rows \#23-24 of table 2). Results obtained for the two samples with different cleaning procedures are compatible; upper limits derived for the sample cleaned using Alconox are more stringent thanks to the larger number of screened units. Once the original grease on the screws is removed, vacuum grease must be used; a sample of Apiezon ${ }^{18} \mathrm{M}$ grease designed for high vacuum applications was analyzed and only upper limits were set (rows \#25 of table 2). Due to the relatively large contribution of these M4 screws (quoted for reference in table 4) the use of the much more radiopure brass bolts is foreseen in the NEXT-100 detector.

Two types of copper supplied by Lugand Aciers company ${ }^{19}$ were screened for use at the energy plane: $\mathrm{CuA} 1^{20}$ for PMT enclosures and base caps and $\mathrm{CuC}^{21}$ for both the tracking and energy readout plates. The weight of each PMT copper enclosure is $4.1 \mathrm{~kg}$ and that of the energy plate $475.6 \mathrm{~kg}$. A large mass of CuA1 copper was accumulated to carry out a measurement using a germanium detector at LSC; a special cleaning procedure typically used for copper shieldings was performed at LSC before the measurement, consisting of soap cleaning, nitric acid etching, passivation with citric acid and drying. As shown in row \#26 of table 2, only ${ }^{60} \mathrm{Co}$ activity was quantified. Peaks from other cobalt isotopes (also common cosmogenic products in copper induced by the exposure of the material to cosmic nucleons at sea level) were identified $\left({ }^{56} \mathrm{Co}\right.$ with $\mathrm{T}_{1 / 2}=77.27 \mathrm{~d}$ and ${ }^{58} \mathrm{Co}$ with $\mathrm{T}_{1 / 2}=70.86 \mathrm{~d}$ ); since their half-lives are of the order of the live time in the screening measurement, the direct quantification of their activities was not performed. GDMS

\footnotetext{
${ }^{17} \mathrm{http}: / /$ alconox.com

$18 \mathrm{http} / / /$ www.apiezon.com

${ }^{19} \mathrm{http}: / /$ lugand-aciers.fr

${ }^{20}$ This type of copper is also referred as Cu-ETP (Electrolytic Tough Pitch) or C11000. Its copper purity is $99.90 \%$ (minimum).

${ }^{21}$ This type of copper is also referred as Cu-OF (Oxygen-Free) or C10200. Its copper purity is $99.95 \%$.
} 
analysis was additionally made for two samples, with dimensions $12 \times 12 \times 12 \mathrm{~mm}^{3}$, made of CuA1 and $\mathrm{CuC} 1$ copper, having received the same cleaning protocol. Following results presented in rows \#27-28 of table 2, GDMS upper limits for the CuA1 sample are much lower than the ones derived from germanium spectrometry in \#26. The good results obtained for this copper led to it being used also for the shielding against gamma radiation to be placed inside the pressure vessel made of 316Ti stainless steel. Results for $\mathrm{CuA} 1$ from Lugand Aciers, at the level of a few $\mu \mathrm{Bq} / \mathrm{kg}$ for ${ }^{238} \mathrm{U}$ and ${ }^{232} \mathrm{Th}$, are equivalent to those obtained for $\mathrm{C} 10100$ copper supplied by the Luvata company [20] and similar to those for the Norddeutsche Affinerie ${ }^{22}$ copper [43]. Results at or even below tenths of $\mu \mathrm{Bq} / \mathrm{kg}$ have been presented for electroformed and also commercial copper analyzed by ICPMS for the Majorana experiment [44, 45].

A sample of the CuSn braid used to dissipate heat at the PMT cans was measured using a germanium detector. It is soft tinned copper wire braid 2536P provided by RS. The total length of the sample was $19.4 \mathrm{~m}$. Upper limits were set for all the common radioisotopes (row \#29 of table 2).

Finally, the expected contribution to the background level in the region of interest of NEXT100, assuming a NEW-like design with 60 PMT modules, from the activities of all the relevant components of the energy plane has been evaluated by Monte Carlo simulation using the Geant 4 package (see details at [18]) and is reported in table 4. A first estimate of the contribution of the energy plane was already made in [18], considering the main components (PMTs, PMT enclosures and sapphire windows). Using the upper limits or the quantified activity of ${ }^{208} \mathrm{Tl}$ and ${ }^{214} \mathrm{Bi}$ presented here for all the selected components for the energy readout, the expected rate in the region of interest for the neutrinoless double beta decay of ${ }^{136} \mathrm{Xe}$ is below $2.4 \times 10^{-4}$ counts $\mathrm{keV}^{-1} \mathrm{~kg}^{-1} \mathrm{y}^{-1}$, complying with the requirements to achieve the desired sensitivity. As it can be concluded from table 4, PMTs and base capacitors are the dominant contributors accounting for $29.9 \%$ and $36.5 \%$, respectively, of the total estimated background.

Apart from the bulk emissions from the measured activity, radon-induced background from surface deposition or emanated radon, as an intermediate decay product of the uranium and thorium series, can be a concern when requiring ultra-low background conditions. Radon can emanate from detector components and be transported to the active volume through the gas circulation. The progeny of radon is positively charged and adhere to surfaces or dust particles; it drifts toward the TPC cathode and the subsequent ${ }^{214} \mathrm{Bi}$ and ${ }^{208} \mathrm{Tl}$ decays are a potential background source. Radon contamination in the xenon gas causes two different types of background events: $\beta$ tracks from the decay of ${ }^{214} \mathrm{Bi}$ in the active volume, and photoelectrons generated by gamma rays emitted, for the most part, from the TPC cathode. This background source was carefully analyzed in [18], evaluating the corresponding background rate generated in NEXT-100 in terms of the activity of ${ }^{222} \mathrm{Rn}$; it was concluded that in order for this background to contribute, at most, at the level of $10^{-5} \mathrm{keV}^{-1} \mathrm{~kg}^{-1} \mathrm{y}^{-1}$, radon activities in the xenon gas below a few $\mathrm{mBq}$ per cubic meter would be required. The design of NEXT-100 minimizes the use of materials and components known to emanate radon in high rates, such as plastics, cables or certain seals and cleaning of surfaces close to the active volume is foreseen. In addition, radon emanation measurements are being carried out for different components used, including those of the energy readout plane, in collaboration with the Jagiellonian University (Cracow, Poland) using a cryogenic radon detector; preliminary results

${ }^{22}$ Re-branded as Aurubis, http://www.aurubis.com 
Table 4. Summary of the expected background rate in the region of interest for NEXT-100 assuming a NEW-like design from the most relevant components of the energy readout plane, estimated by Monte Carlo simulation. Activities considered are indicated in the second column, either from some references or from entries in table 2. Upper limits are at $95.5 \%$ C.L. All numbers correspond to $10^{-4}$ counts $\mathrm{keV}^{-1} \mathrm{~kg}^{-1} \mathrm{y}^{-1}$. Contribution marked with (*) is not included in the total sum (see text). As a reference, the estimate of the background rate from the energy plane in [18] considering just PMTs, PMT enclosures and sapphire windows was $1.0 \times 10^{-4}$ counts $\mathrm{keV}^{-1} \mathrm{~kg}^{-1} \mathrm{y}^{-1}$.

\begin{tabular}{lccc}
\hline Component & Activity & Rate from ${ }^{208} \mathrm{Tl}$ & Rate from ${ }^{214} \mathrm{Bi}$ \\
\hline PMTs & $\# 2$ & $0.35 \pm 0.08$ & $0.37 \pm 0.08$ \\
Base capacitors $1.5 \mu \mathrm{F}$ & $\# 3$ & $0.125 \pm 0.007$ & $0.377 \pm 0.016$ \\
Base capacitors $4.7 \mu \mathrm{F}$ & $\# 4$ & $0.113 \pm 0.008$ & $0.258 \pm 0.015$ \\
Finechem base resistors & $\# 6$ & $0.040 \pm 0.003$ & $0.060 \pm 0.004$ \\
RS base resistors & $\# 7$ & $<0.013$ & $<0.040$ \\
Pin receptacles at base & $\# 9$ & $0.053 \pm 0.005$ & $<0.02$ \\
Epoxy Araldite & $\# 11$ & $<0.014$ & $<0.012$ \\
Kapton-Cu cable & $\# 13$ & $<0.006$ & $0.010 \pm 0.001$ \\
Kapton substrate & $\# 15$ & $0.036 \pm 0.006$ & $<0.030$ \\
Copper caps at base & $\# 27$ & $<110^{-4}$ & $<610^{-4}$ \\
Sapphire windows & Ref. [40] & $0.018 \pm 0.004$ & $<0.11$ \\
Optical gel & $\# 17$ & $<0.041$ & $<0.13$ \\
TPB & Ref. [42] & $(1.3 \pm 0.3) 10^{-5}$ & $(1.1 \pm 0.4) 10^{-4}$ \\
PEDOT:PSS & $\# 19$ & $<2.610^{-6}$ & $<9.410^{-6}$ \\
Brazing paste & $\# 20$ & $(2.1 \pm 0.2) 10^{-4}$ & $(6.0 \pm 1.1) 10^{-4}$ \\
M4 screws $(*)$ & $\# 24$ & $0.52 \pm 0.05$ & $<0.66$ \\
Brass bolts & $\# 21$ & $(6.4 \pm 0.2) 10^{-4}$ & $(2.1 \pm 0.2) 10^{-3}$ \\
PMT copper enclosures & $\# 27$ & $<0.001$ & $<0.002$ \\
Copper plate & $\# 28$ & $0.054 \pm 0.014$ & $0.065 \pm 0.013$ \\
CuSn braid & $\# 29$ & $<0.014$ & $<0.025$ \\
\hline Total & & $<0.88$ & $<1.51$ \\
\hline & & & \\
\hline
\end{tabular}

point to acceptable activities of ${ }^{220} \mathrm{Rn}$ and ${ }^{222} \mathrm{Rn}$ for the PMTs, the TPB-coated PMT windows and the kapton-copper cable.

\section{Conclusion}

A thorough control of the material radiopurity is being performed in the construction of the NEXT double beta decay experiment to be operated at LSC, mainly based on activity measurements using ultra-low background gamma-ray spectrometry with germanium detectors of the Radiopurity Service of LSC and complementary GDMS analysis. Radiopurity information is helpful not only for the selection of sufficiently radiopure materials, but also for the development of the detector background model in combination with Monte Carlo simulations. Many of the components to be actually used in the experiment, like the PMT units, have been directly screened. 
The design of a radiopure energy readout plane for the NEXT detection system, which must be in direct contact with the gas detector medium, was a challenge (as it was for the tracking plane [27]) since photomultipliers and electronic components can typically have much higher activity levels than those tolerated in ultra-low background experiments. Selection of in-vessel components was performed in parallel to its design.

Photomultiplier tubes are the main element of the energy readout in the NEXT detector. All the available units of the selected PMT model, Hamamatsu R11410-10, were screened in 3-unit groups using the same germanium detector at LSC. Compatible activities were registered for all runs and a joint analysis of all the accumulated data allowed us to quantify average activities of not only ${ }^{60} \mathrm{Co}$ and ${ }^{40} \mathrm{~K}$ but also of the isotopes in the lower part of the ${ }^{238} \mathrm{U}$ and ${ }^{232} \mathrm{Th}$ chains, of uppermost relevance for NEXT-100 background. The found activities are similar to those measured for the new version of the PMT R11410-21 [34], except for ${ }^{60} \mathrm{Co}$, which is about five times larger in the version considered here. In addition, most of the components accompanying PMTs at their bases and enclosures were analyzed, including sapphire windows, optical gel, capacitors, resistors, cables, epoxy, bolts, screws and copper; results are summarized in table 2. The procurement of large samples with a huge number of pieces made it possible to measure activities at the level of $\mu \mathrm{Bq} / \mathrm{unit}$ for several components. Some items were disregarded due to bad radiopurity and replaced by other ones.

The construction of a precise NEXT-100 background model is based on Geant 4 simulation and it allows us to evaluate the experimental sensitivity. After the first estimate made in [18], the contribution from the energy plane to the background level in the region of interest for the neutrinoless double beta decay of ${ }^{136} \mathrm{Xe}$ has been reanalyzed here considering all the material radiopurity information collected and assuming a NEW-like design; as shown in table 4, PMTs and base capacitors are found to be the dominant contributors. The expected rate is below $2.4 \times$ $10^{-4}$ counts $\mathrm{keV}^{-1} \mathrm{~kg}^{-1} \mathrm{y}^{-1}$, satisfying the requirements to achieve the desired sensitivity. But this contribution could be further reduced thanks to some changes implemented in the PMT bases design or other components for the final NEXT-100 detector.

\section{Acknowledgments}

Special thanks are due to LSC directorate and staff for their strong support for performing the measurements at the LSC Radiopurity Service. We are really grateful to Grzegorz Zuzel for the radon emanation measurements. The NEXT Collaboration acknowledges support from the following agencies and institutions: the European Research Council (ERC) under the Advanced Grant 339787-NEXT; the Ministerio de Economía y Competitividad of Spain under grants FIS2014-53371-C04 and the Severo Ochoa Program SEV-2014-0398; the GVA of Spain under grant PROMETEO/2016/120; the Portuguese FCT and FEDER through the program COMPETE, project PTDC/FIS/103860/2008; the U.S. Department of Energy under contracts number DE-AC0207CH11359 (Fermi National Accelerator Laboratory) and DE-FG02-13ER42020 (Texas A\&M); and the University of Texas at Arlington. 


\section{References}

[1] J.J. Gomez-Cadenas et al, The search for neutrinoless double beta decay, Riv. Nuovo Cim. 35 (2012) 29-98.

[2] S. R. Elliott, Recent Progress in Double Beta Decay, Mod. Phys. Lett. A 27 (2012) 1230009.

[3] F. T. Avignone III et al., Double Beta Decay, Majorana Neutrinos, and Neutrino Mass, Rev. Mod. Phys. 80 (2008) 481.

[4] O. Cremonesi and M. Pavan, Challenges in Double Beta Decay, Advances in High Energy Physics 2014 (2014) 951432.

[5] J. J. Gomez-Cadenas et al, Present Status and Future Perspectives of the NEXT Experiment, Advances in High Energy Physics 2014 (2014) 907067.

[6] http://www.lsc-canfranc.es

[7] V. Alvarez et al., Near-Intrinsic Energy Resolution for 30 to 662 keV Gamma Rays in a High Pressure Xenon Electroluminescent TPC, Nucl. Instrum. Meth. A 708 (2013) 101-114.

[8] V. Alvarez et al., Initial results of NEXT-DEMO, a large-scale prototype of the NEXT-100 experiment, JINST 8 (2013) P04002.

[9] V. Alvarez et al., Ionization and scintillation response of high-pressure xenon gas to alpha particles, JINST 8 (2013) P05025.

[10] V. Alvarez et al., Operation and first results of the NEXT-DEMO prototype using a silicon photomultiplier tracking array, JINST 8 (2013) P09011.

[11] V. Alvarez et al., Description and commissioning of NEXT-MM prototype: first results from operation in a Xenon-Trimethylamine gas mixture, JINST 9 (2014) P03010.

[12] V. Alvarez et al., Characterization of a medium size Xe/TMA TPC instrumented with microbulk Micromegas, using low-energy $\gamma$-rays, JINST 9 (2014) C04015.

[13] D. Lorca et al., Characterisation of NEXT-DEMO using xenon $K_{\alpha}$ X-rays, JINST 9 (2014) P10007.

[14] D. Gonzalez-Diaz et al., Accurate $\gamma$ and MeV-electron track reconstruction with an ultra-low diffusion Xenon/TMA TPC at 10 atm, Nucl. Instrum. Meth. A 804 (2015) 8-24.

[15] J. Renner et al., Ionization and scintillation of nuclear recoils in gaseous xenon, Nucl. Instrum. Meth. A 793 (2015) 62-74.

[16] L. Serra eta al., An improved measurement of electron-ion recombination in high-pressure xenon gas, JINST 10 (2015) P03025.

[17] P. Ferrario et al., First proof of topological signature in the high pressure xenon gas TPC with electroluminescence amplification for the NEXT experiment, JHEP 01 (2016) 104.

[18] J. Martin-Albo et al., Sensitivity of NEXT-100 to neutrinoless double beta decay, JHEP 05(2016) 159.

[19] J. Renner et al., Background rejection in NEXT using deep neural networks, JINST 12 (2017) T01004.

[20] V. Alvarez et al., Radiopurity control in the NEXT-100 double beta decay experiment: procedures and initial measurements, JINST 8 (2013) T01002.

[21] V. Alvarez et al., Radiopurity control in the NEXT-100 double beta decay experiment, AIP Conf. Proc. 1549 (2013) 46.

[22] T. Dafni et al., Results of the material screening program of the NEXT experiment, Nucl. Part. Phys. Proc. 273-275 (2016) 2666-2668. 
[23] S. Cebrian et al., Radon and material radiopurity assessment for the NEXT double beta decay experiment AIP Conf. Proc. 1672 (2015) 060002.

[24] G. Heusser, Low-radioactivity background techniques, Annu. Rev. Nucl. Part. Sci. 45 (1995) 543-590.

[25] ILIAS Database, http://radiopurity.in2p3.fr.

[26] J.C. Loach et al., A Database for Storing the Results of Material Radio-purity Measurements, Nucl. Instrum. Meth. A 839 (2016) 6-11.

[27] S. Cebrian et al., Radiopurity assessment of the tracking readout for the NEXT double beta decay experiment, JINST 10 (2015) P05006.

[28] I. Bandac et al., Ultra-Low Background and Environmental Measurements at Laboratorio Subterráneo de Canfranc (LSC), Appl. Rad. and Isot. (2017) in press.

[29] S. Agostinelli et al., GEANT4-a simulation toolkit, Nucl. Instrum. Meth. A 506 (2003) 250.

[30] E. Aprile et al., Material screening and selection for XENON100, Astropart. Phys. 35 (2011) $43-49$.

[31] D.S. Akerib et al., An Ultra-Low Background PMT for Liquid Xenon Detectors, Nucl. Instrum. Meth. A 703 (2013) 1-6.

[32] X.G. Cao et al., PandaX: A Liquid Xenon Dark Matter Experiment at CJPL, SCIENCE CHINA Physics, Mechanics \& Astronomy 57 (2014) 1476-1494. X. Wang et al., Material Screening with HPGe Counting Station for PandaX Experiment, JINST 11 (2016) T12002.

[33] P. Barrow et al., Qualification Tests of the R11410-21 Photomultiplier Tubes for the XENON1T Detector, JINST 12 (2017) P01024.

[34] E. Aprile et al., Lowering the radioactivity of the photomultiplier tubes for the XENONIT dark matter experiment, Eur. Phys. J. C 75 (2015) 546.

[35] E. Aprile et al., Material radioassay and selection for the XENON1T dark matter experiment, arXiv:1705.01828v1 [physics.ins-det].

[36] The LUX-ZEPLIN collaboration, The LUX-ZEPLIN (LZ) Technical Design Report, arXiv:1703.09144v1 [physics.ins-det].

[37] J. Busto et al., Radioactivity measurements of a large number of adhesive, Nucl. Instrum. Meth. A 492 (2002) 35-42.

[38] D. Budjas et al., Gamma-ray spectrometry of ultra low levels of radioactivity within the material screening program for the GERDA experiment, Appl. Rad. and Isot. 67 (2009) 755.

[39] S. Nisi et al., Comparison of inductively coupled mass spectrimetry and ultra low-level gamma-ray spectroscopy for ultra low background material selection, Appl. Rad. and Isot. 67 (2009) 828.

[40] S. Leonard et al., Systematic study of trace radioactive impurities in candidate construction materials for EXO-200, Nucl. Instrum. Meth. A 591 (2008) 490. D.S. Leonard et al., Trace radioactive impurities in final construction materials for EXO-200, arXiv:1703.10799v1 [physics.ins-det].

[41] I. Lawson and B. Cleveland, Low Background Counting At SNOLAB, AIP Conf. Proc. 1338 (2011) 68-77.

[42] M.L. di Vacri et al., ICP MS selection of radiopure materials for the GERDA experiment AIP Conf. Proc. 1672 (2015) 150001.

[43] M. Laubenstein et al., Underground measurements of radioactivity, Appl. Rad. and Isot. 61 (2004) 167-172. 
[44] B.D. LaFerriere et al., A novel assay method for the trace determination of $T$ Th and $U$ in copper and lead using inductively coupled plasma mass spectrometry, Nucl. Instrum. Meth. A 775 (2015) 93-98.

[45] N. Abgrall et al., The Majorana Demonstrator Radioassay Program, Nucl. Instrum. Meth. A 828 (2016) 22-36. 